\title{
Continuous-Curvature Path Generation Using Fermat's Spiral
}

\author{
Anastasios M. Lekkas ${ }^{1}$ Andreas R. Dahl ${ }^{2}$ Morten Breivik $^{3}$ Thor I. Fossen ${ }^{4}$ \\ ${ }^{1}$ Centre for Ships and Ocean Structures, Norwegian University of Science and Technology, NO-7491 Trondheim, \\ Norway. E-mail: anastasios.lekkas@itk.ntnu.no \\ ${ }^{2}$ Department of Marine Technology, Norwegian University of Science and Technology, NO-7491 Trondheim, \\ Norway. E-mail: andreas.r.dah/@ntnu.no \\ ${ }^{3}$ Department of Engineering Cybernetics, Norwegian University of Science and Technology, NO-7491 Trondheim, \\ Norway. E-mail: morten.breivik@ieee.org \\ ${ }^{4}$ Centre for Autonomous Marine Operations and Systems, Department of Engineering Cybernetics, Norwegian \\ University of Science and Technology, NO-7491 Trondheim, Norway. E-mail: fossen@ieee.org
}

\begin{abstract}
This paper proposes a novel methodology, based on Fermat's spiral (FS), for constructing curvaturecontinuous parametric paths in a plane. FS has a zero curvature at its origin, a property that allows it to be connected with a straight line smoothly, that is, without the curvature discontinuity which occurs at the transition point between a line and a circular arc when constructing Dubins paths. Furthermore, contrary to the computationally expensive clothoids, FS is described by very simple parametric equations that are trivial to compute. On the downside, computing the length of an FS arc involves a Gaussian hypergeometric function. However, this function is absolutely convergent and it is also shown that it poses no restrictions to the domain within which the length can be calculated. In addition, we present an alternative parametrization of FS which eliminates the parametric speed singularity at the origin, hence making the spiral suitable for path-tracking applications. A detailed description of how to construct curvature-continuous paths with FS is given.
\end{abstract}

Keywords: Path planning, Fermat's spiral, continuous curvature, parametric curve, path tracking

\section{Introduction}

Path-planning systems are of great significance when it comes to the performance and mission accomplishment of practically every type of vehicle, as well as mechatronic devices such as computer numerical control (CNC) machines and robotic manipulators, to name a few. Depending on the task demands, the path-planning algorithms generate appropriate paths or trajectories usually by taking into account both the physical constraints of the system under consideration, and the workspace constraints, such as obstacles or en- vironmental forces. A thorough treatment of the subject can be found in (LaValle, 2006).

In the case of underactuated vehicles, especially in path-following and path-tracking motion control scenarios, the shape and properties of the path have a direct influence on the guidance system, which is responsible for generating reference trajectories to be fed to the autopilot. The reason for this is that the path curvature affects the heading angle commands and the parametric speed affects the velocity commands. In these applications, as a rule, the path-planning algorithm will first define a number of ordered waypoints 
on the map which will have to be connected sequentially so as to form the path. Connecting the waypoints can be achieved in many different ways, with each one having its own advantages and drawbacks. However two main categories can be distinguished:

- Combining straight lines and arc segments.

- Using splines.

Regarding the first category, a simple and intuitive way of getting continuous paths without corners is to inscribe a circle between two lines in order to form a curved path. In 1957, Dubins showed that for a particle that moves forward with unity speed, the shortest possible path that meets a maximum curvature bound between a starting position with predefined orientation (starting pose) and a finishing position with predefined orientation (finishing pose) consists of at most three pieces, each of which is either a straight line or an arc of a circle of radius $R>0$ (Dubins, 1957). Reeds and Shepp (1990) extended Dubins' result for a car-like vehicle by taking into account backward motion, hence allowing to include cusps along the path. The Dubins car was further extended to include altitude, hence leading to the Dubins airplane (Chitsaz and LaValle, 2007). A discrete analogue of Dubins' result using polygonal paths was proved by Eriksson-Bique et al. (2012), although the authors state that different definitions of discrete curvature-constrained motion than the one they used might lead to shorter paths. Chang et al. (2012) augmented Dubins' result by incorporating a directional-cost element in order to minimize the costs associated with the construction of underground mines. In (Hota and Ghose, 2013), a modified Dubins problem was considered where a rectilinear path and direction, to which a vehicle should converge, was specified instead of a final point. The goal was to find the optimal 2D path for convergence to a rectilinear path for a vehicle that is initially located at any distance from the desired path. Moreover, Techy and Woolsey (2009), Bakolas and Tsiotras (2010) and Bakolas and Tsiotras (2013) deal with the problem of finding a Dubins path for a vehicle that moves in a constant drift field. In this case, it is not always possible to find a Dubins path.

The main disadvantage of the Dubins path is the curvature discontinuity which occurs at the meeting points of two consecutive path segments, for more details see (Tsourdos et al., 2011). This problem can be circumvented by employing a clothoid arc between a straight line and a circular arc (Fraichard and Scheuer, 2004). The clothoid (also known as Euler spiral, Cornu spiral and spiro) is useful in path-planning applications due to its property of having its curvature change linearly with arc-length. This notion can be extended to three dimensions, and is consequently also true for the torsion (Harary and Tal, 2012). Clothoids have also been used in formation control applications, see for instance (Shanmugavel et al., 2007a) and (Shanmugavel et al., 2010). The drawback of clothoids is that their coordinates do not have a closed-form expression since they involve computation of the Fresnel integrals.

The second category of methodologies pertains to connecting waypoints using splines. There is a vast literature on this subject, mostly due to the research stemming from the computer graphics community. Some approaches, such as the cubic Hermite spline interpolation (CHSI), result in continuous velocity paths with more tractable shape (without wiggling between the waypoints) but have a discontinuous curvature at the waypoint locations. Others, like the natural cubic splines, give curvature-continuous paths but the resulting shape can be very impractical and inefficient. An interesting alternative is to use Pythagoreanhodograph $(\mathrm{PH})$ curves which are characterized by the special property that their parametric speed is a polynomial (or rational) function of the path parameter (Farouki, 2008). The PH curves were presented for the first time in (Farouki and Sakkalis, 1990) and the idea occurred while the authors were investigating the existence of planar or spatial curves that have natural parametrizations (Farouki and Sakkalis, 1991). PH curves do not necessarily entail curvaturecontinuous paths, this issue was addressed by Bruyninckx and Reynaerts (1997). In addition, several other researchers have employed $\mathrm{PH}$ curves for path planning, see for instance (Toussaint et al., 2001), (Shanmugavel et al., 2007b) and (Gajny et al., 2012). Interestingly, $\mathrm{PH}$ curves of monotone curvature have also been used to connect straight lines in a way such that the overall curve is curvature continuous (Farouki, 1997).

Contrary to what might be the case in the field of computer graphics, where often the goal is to design aesthetically pleasing curves (Farin and Sapidis, 1989), in motion control scenarios it is preferable to assign paths that consist of straight lines and arc segments rather than splines, mainly because the latter implies that at least one of the control surfaces (a ship's rudder, for instance) will always be active, due to the relentlessly varying curvature. In addition, using straight lines and arc segments makes it more likely and easier to ensure that the path will not include wiggles or zigzags between two waypoints. This property, which is also related to allowance (Dahl, 2013), can be critical when navigating in an area where obstacles are present. Last but not least, moving on straight lines enables adaptive techniques to provide a faster and more robust estimate of unknown external disturbances, such as ocean currents (Fossen and Lekkas, 2014). 
This paper proposes an alternative way of designing curvature-continuous paths, which belongs to the first of the two aforementioned categories. More specifically, Fermat's spiral (FS) is employed as a means to connect successive straight lines in a plane. The main motivation is that the curvature of FS is equal to zero at the origin, a property which makes it suitable for connecting it with a straight line without inducing curvature discontinuities, as is the case with circular arcs. Moreover, contrary to clothoids, FS involves very simple parametric equations that are easy to program and fast to compute. The use of FS for pathplanning applications was initially studied by Dahl (2013) and later implemented for building curvaturecontinuous and collision-free paths using Voronoi diagrams in (Candeloro et al., 2013). In this paper, we investigate the properties of FS and show that it can accomplish the task of generating curvature-continuous paths and therefore be used in path-following applications. Furthermore, we extend the work done by Dahl (2013) and Candeloro et al. (2013) in three ways: a) we propose a different parametrization which removes the speed singularity at the origin, hence making FS paths suitable also for path-tracking applications, b) we present a more thorough analysis regarding the calculation of the FS arc length, and c) we show that FS can be used successfully in combination with circular arcs in order to produce Dubins paths with FS arc transition.

\section{Preliminaries}

\subsection{Basic Definitions}

Similarly to Breivik and Fossen (2009), a planar path is considered to be a one-dimensional manifold that can be expressed by the set

$$
\mathcal{P}:=\left\{\mathbf{p} \in \mathbb{R}^{2} \mid \mathbf{p}=\mathbf{p}_{p}(\varpi) \forall \varpi \in \mathbb{R}\right\}
$$

where $\varpi$ is the path parameter and $\mathbf{p}_{p}(\varpi)$ denotes the position of a point belonging to the path. The generalized path parameter does not necessarily have any physical meaning. It can be the path length, but is usually more convenient to be defined within the unit domain:

$$
\varpi \in[0,1]
$$

because it can be difficult to construct an elegant basis for a general interval $\left[\varpi_{i}, \varpi_{i+1}\right]$. It is common practice to implement $\mathbf{p}(\cdot)$ as a piecewise-defined function, which reduces the function complexity but demands consideration at the transition points between subpaths. For planar paths consisting of a number of curve segments, each single curve segment can be expressed by the set (Haugen, 2010):

$$
\begin{aligned}
\mathcal{P}_{i}:=\left\{\mathbf{p}_{i}\right. & \in \mathbb{R}^{2} \mid \\
\mathbf{p}_{i} & \left.=\mathbf{p}_{i, p}(\varpi) \forall \varpi \in \mathcal{I}_{i}=\left[\varpi_{i, 0}, \varpi_{i, 1}\right] \subset \mathbb{R}\right\},
\end{aligned}
$$

and, consequently, the path can be written as a superset of $n$ curve segments:

$$
\mathcal{P}_{s}=\bigcup_{i=1}^{n} \mathcal{P}_{i}
$$

In this paper, we consider two-dimensional curves:

$$
\mathbf{p}_{p}(\varpi)=\left[\begin{array}{l}
x_{p}(\varpi) \\
y_{p}(\varpi)
\end{array}\right],
$$

from which, the path-tangential (or, alternatively, course) angle is computed as:

$$
\chi_{p}(\varpi)=\operatorname{atan} 2\left(y_{p}^{\prime}(\varpi), x_{p}^{\prime}(\varpi)\right),
$$

where $(\cdot)^{\prime}$ denotes the first derivative w.r.t the path parameter $\varpi$. In contrast, the derivation w.r.t. time is denoted $(\cdot)$. The parametric path $(5)$ is regular when:

$$
\left|\mathbf{p}_{p}^{\prime}(\varpi)\right|=\sqrt{\left(x_{p}^{\prime}(\varpi)\right)^{2}+\left(y_{p}^{\prime}(\varpi)\right)^{2}} \neq 0 .
$$

The curvature of the regular path is given by the expression:

$$
\kappa=\frac{x_{p}^{\prime}(\varpi) y_{p}^{\prime \prime}(\varpi)-y_{p}^{\prime}(\varpi) x_{p}^{\prime \prime}(\varpi)}{\sqrt[3]{\left(x_{p}^{\prime}(\varpi)\right)^{2}+\left(y_{p}^{\prime}(\varpi)\right)^{2}}}
$$

Naturally, a minimum requirement is that subsequent path segments should be connected. The level of connection can be described by the path smoothness. Parametric continuity is denoted $C^{n}$, where $n$ is the degree of smoothness, and implies that the first $n$ parametric derivatives match at the common point of two subsequent paths (Barsky and DeRose, 1989). Similarly, geometric continuity is denoted $G^{n}$, and is a relaxed form of parametric continuity, which does not impose constraints on the parameter's speed along the path. For many applications (such as path following of underactuated vehicles) it is sufficient to have geometric continuity, while for others (such as path tracking) parametric continuity is required. For more information on path smoothness as well as other path evaluation criteria, the reader is referred to Dahl (2013) and Lekkas and Fossen (2013).

Given a number of ordered points on a plane, it is useful to distinguish the following two types of curves:

- Interpolating curves, which pass through all the given points. 


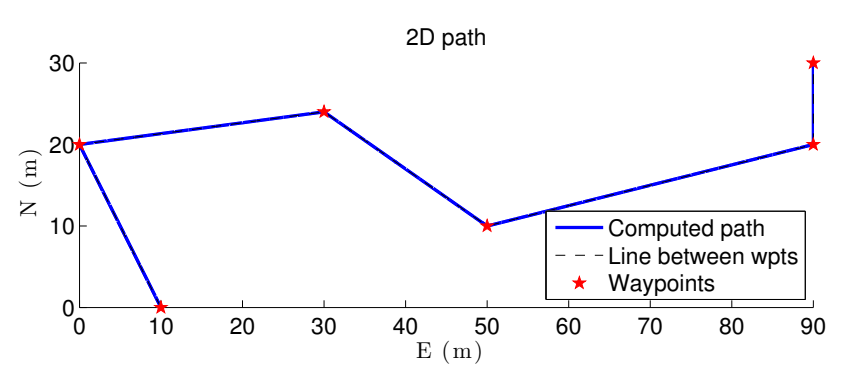

Figure 1: Piecewise linear path
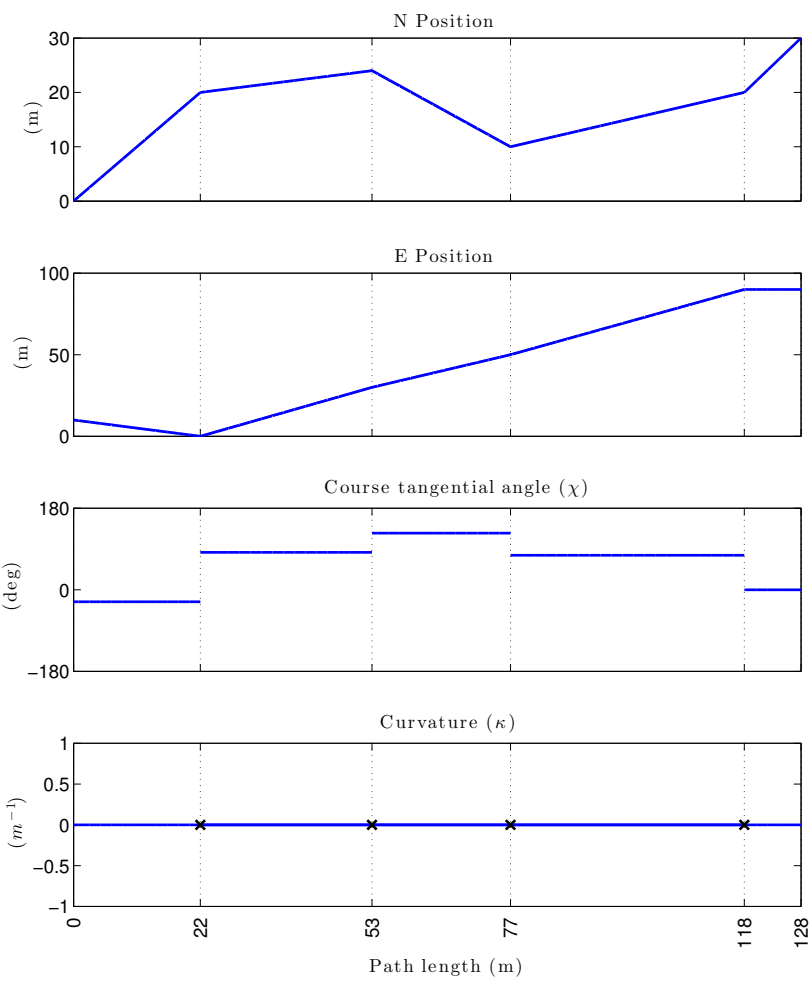

Figure 2: Piecewise linear path geometric properties

- Approximating curves, which do not pass through all the given points.

This categorization helps describe more accurately methods that use the same building blocks but have different properties. For example, both the Dubins paths and the paths resulting from circular smoothing (Fossen, 2011, Sec. 10.3.1) use straight lines and circular arcs, however they result in different paths.

\subsection{Piecewise Linear Path}

The parametric form of a straight line connecting two points in a plane is given by (Breivik and Fossen, 2009):

$$
\mathbf{p}_{\text {line }}(\varpi)=\left[\begin{array}{c}
N_{0}+L \varpi \cos \left(\chi_{l}\right) \\
E_{0}+L \varpi \sin \left(\chi_{l}\right)
\end{array}\right],
$$

where $\mathbf{p}_{0}=\left[N_{0}, E_{0}\right]^{\mathrm{T}}$ is the starting point, $L$ is the length of the path, and $\chi_{l}$ is the path-tangential angle of the straight line. An example of a path consisting only of straight lines can be seen in Fig. 1. As it can be observed in Fig. 2, the path tangential angle is discontinuous and the curvature cannot be defined at the waypoint locations. Such a path cannot be followed by an underactuated vehicle and should only be assigned to a fully-actuated (3-DOF in the planar case) vehicle, such as a wheeled mobile robot. Even in that case, though, the robot must stop at the waypoint locations and then change its heading so as to adjust its attitude according to the direction of the next linear segment before starting to move along it. This problem can be alleviated by using circular segments to smooth the path.

\subsection{Circular Arcs}

Constructing a path using linear and circular arc segments is an extensively studied and popular approach which also leads to the shortest feasible path, according to Dubins' result (Dubins, 1957). Two path variations are probably the most common ones in this case, and their differences occur due to the different locations where the circular segments are placed. The first variation defines an interpolating curve that passes through all the waypoints (that is, the Dubins path), while the second defines an approximating curve that passes only through the first and last waypoint (also known as circular smoothing path), see (Breivik and Fossen, 2004b) and (Fossen, 2011, Sec. 10.3). Both are $G^{1}$ paths, but the final geometry is different. A circular arc can be parametrized as follows:

$$
\mathbf{p}_{\text {cir }}(\varpi)=\left[\begin{array}{c}
c_{N}+R \cos \left(\alpha_{0}+\varpi\left(\alpha_{1}-\alpha_{0}\right)\right) \\
c_{E}+R \sin \left(\alpha_{0}+\varpi\left(\alpha_{1}-\alpha_{0}\right)\right)
\end{array}\right],
$$

where $\mathbf{c}=\left[c_{N}, c_{E}\right]^{\mathrm{T}}$ and $R$ are the center and radius of the circle which the arc is a segment of, while $\alpha_{0}$ and $\alpha_{1}$ are the angles at which the arc starts and ends, respectively. Fig. 3 shows a straight-line path smoothened by circular arcs. The circular arc radius $R$ is chosen equal to that of the vehicle's minimum turning circle (or, the sharpest turn achievable by the vehicle), which corresponds to the curvature constraint:

$$
\kappa_{\max }=\frac{1}{R_{\min }} .
$$

It can be seen in Fig. 4 that the course tangential angle is continuous but the curvature is discontinuous at the locations where the transition between a straight line and a circular arc occurs, which indicates that the path is $G^{1}$. The curvature discontinuity entails a discontinuity in the desired lateral acceleration of the vehicle 


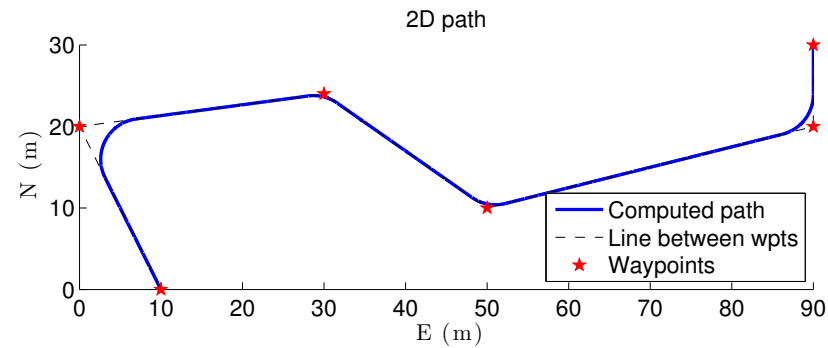

Figure 3: Circular smoothing path
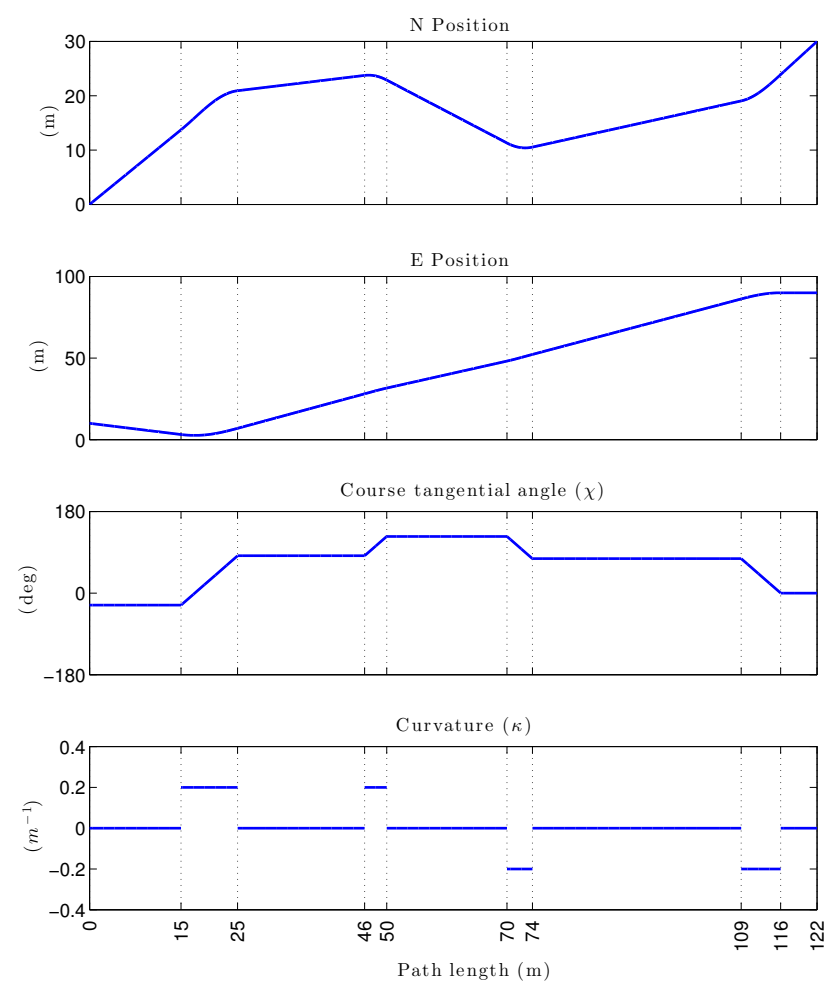

Figure 4: Circular smoothing path geometric properties

because of the relationship (Tsourdos et al., 2011):

$$
|\boldsymbol{\alpha}|=|\mathbf{u}|^{2} \kappa
$$

where $\boldsymbol{\alpha}$ is the lateral acceleration vector and $\mathbf{u}$ the velocity vector. This will affect the input to the heading controller and the vehicle performance in general. Finally, from Figs 2 and 4 it can be observed that the path using circular arcs is shorter. This agrees with intuition since the circular arc operates as a shortcut and avoids going all the way to the second waypoint.

\subsection{Clothoid}

The clothoid is a curve with linear increase in curvature along its length and thus an appealing transition curve.

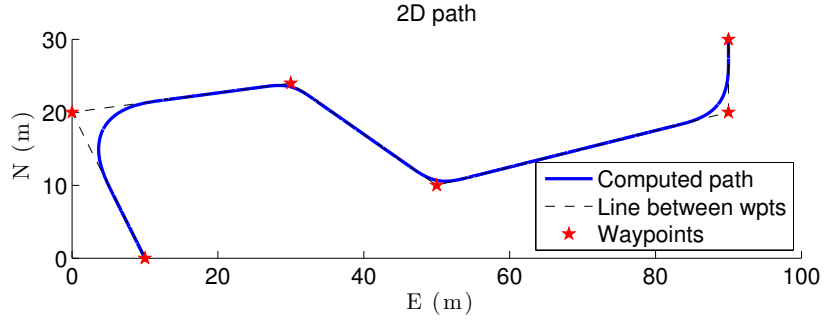

Figure 5: Clothoid smoothing path
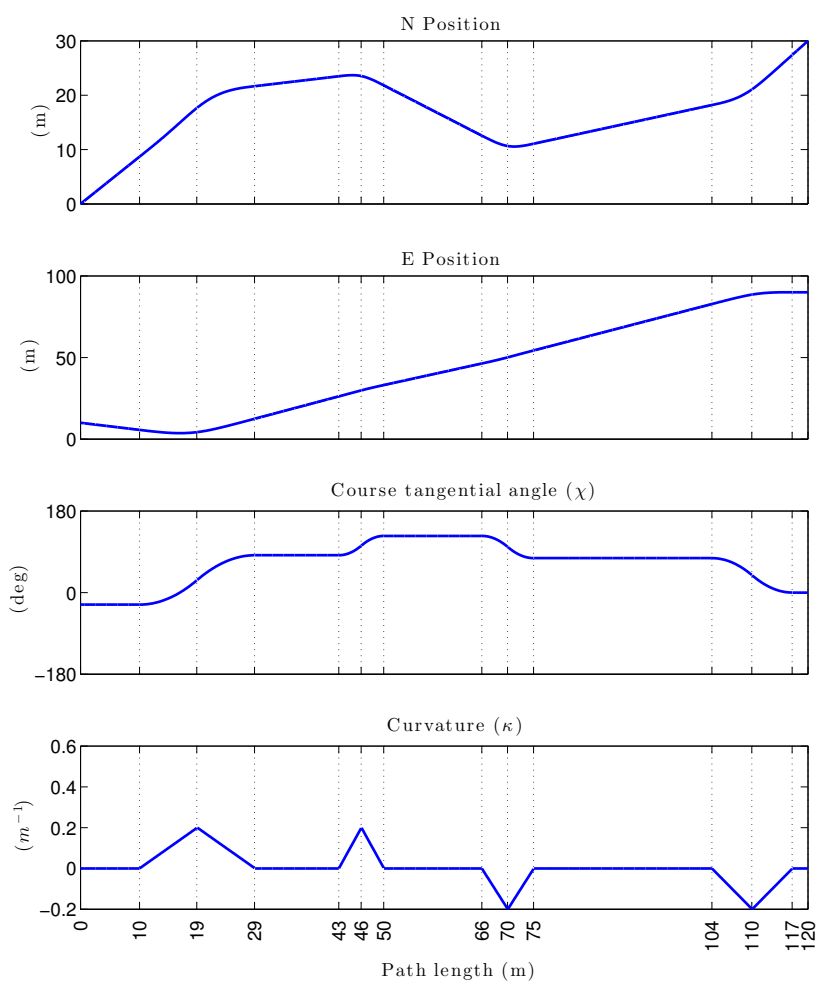

Figure 6: Clothoid smoothing path geometric properties

Its parametrization is

$$
\mathbf{p}_{\mathrm{cl}}(\varpi)=\left[\begin{array}{c}
x_{0}+\int_{0}^{\varpi} \cos \left(\frac{1}{2} c \xi^{2}+\kappa_{0} \xi+\chi_{0}\right) d \xi \\
y_{0}+\int_{0}^{\varpi} \sin \left(\frac{1}{2} c \xi^{2}+\kappa_{0} \xi+\chi_{0}\right) d \xi
\end{array}\right],
$$

where $\mathbf{p}_{0}=\left[x_{0}, y_{0}\right]^{\mathrm{T}}$ is the initial position, $c$ is a sharpness coefficient describing the increase in curvature, $\kappa_{0}$ is the curvature at $\mathbf{p}_{0}, \chi_{0}$ is the course angle at $\mathbf{p}_{0}$ and $\xi$ is a dummy integration variable. Unfortunately, the integrals of (13) have no analytic solution and a numerical approach must be employed.

There are two ways clothoids can be used as transition curves. The first one is to replace the circular arc with a clothoid. Since the clothoid has an increasing curvature w.r.t. the path length, it is necessary to use two clothoid segments: one with starting point at 


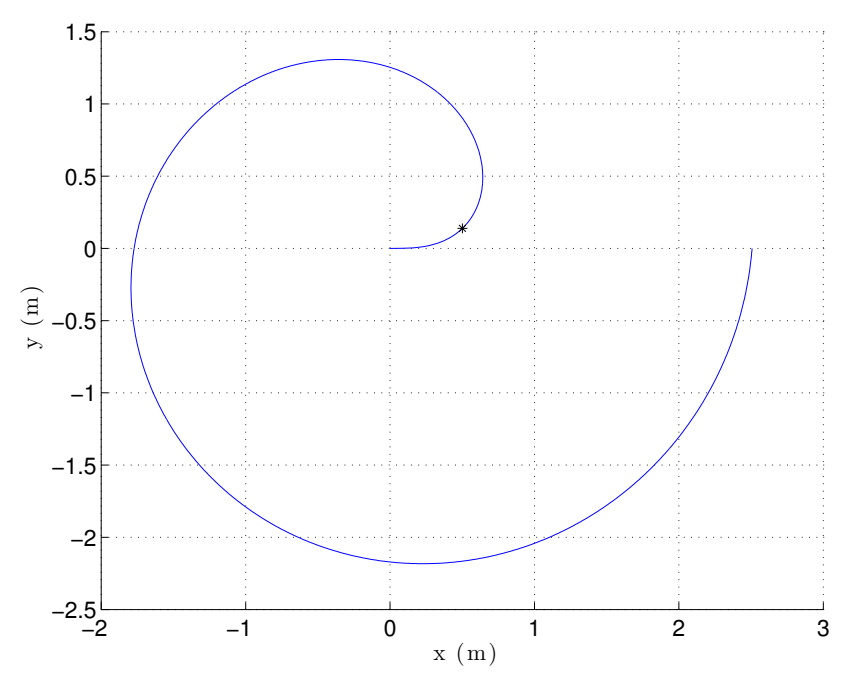

Figure 7: Fermat's spiral, one complete rotation. The asterisk marks the point where the curve has the maximum curvature

the end of the first straight line and ending point at the middle of the curved segment (this is the part with increasing curvature), and one with starting point at the middle of the curved segment and ending point at the beginning of the second straight line (this is the part with decreasing curvature). The second clothoid segment is actually a mirrored version of the first one, therefore the computation needs to be done only once. We will refer to this as clothoid smoothing. The second approach is to use the clothoid as a transition curve between the linear segment and the circular arc. To achieve curvature continuity, that is $G^{2}$ continuity, the transition curve must have zero curvature at one end and $R^{-1}$ at the other. Similarly to clothoid smoothing, a mirrored curve is necessary for the transition between the circular arc and the second straight line. We will refer to this as circular smoothing with clothoid transition. Figs 5-6 show a clothoid smoothing path and its geometrical properties, respectively. In this case, the length of the path is even shorter, when compared to the circular smoothing case. This is related to the different wheel over and pull out points of the arc in each case. In accordance with what has been mentioned earlier, this is an approximating path.

The computational cost of (13) is the main motivation for investigating alternative solutions. One such alternative is Fermat's spiral, and the contribution of this article is the application of such a spiral for path smoothing.

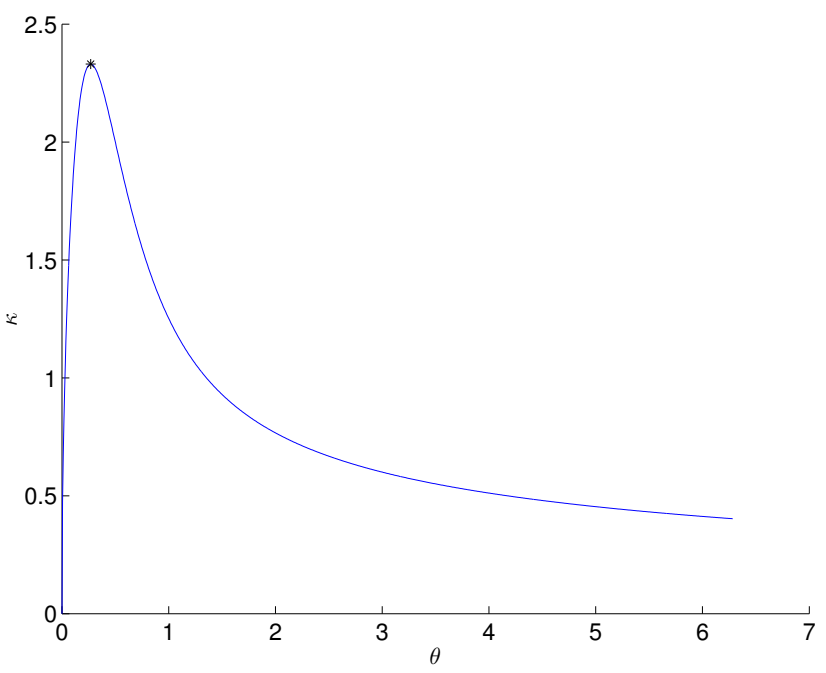

Figure 8: Fermat's spiral curvature. The asterisk marks the value of $\theta$ for which the curve has the maximum curvature.

\section{Fermat's Spiral}

\subsection{Introduction}

The term Archimedean spirals refers to a family of curves described by the equation (Weisstein, 2013a):

$$
r=k \theta^{1 / n},
$$

where $r$ is the radial distance, $\theta$ is the polar angle, $k$ is a scaling constant, and $n$ determines how tightly the spiral is wrapped. The Fermat's spiral (FS) is an Archimedean spiral with $n=2$ and therefore given by the equation:

$$
r=k \sqrt{\theta} .
$$

The spiral curvature is (Weisstein, 2013b):

$$
\kappa(\theta)=\frac{1}{k} \frac{2 \sqrt{\theta}\left(3+4 \theta^{2}\right)}{\left(1+4 \theta^{2}\right)^{\frac{3}{2}}},
$$

which gives $\kappa(0)=0$ and $\kappa(\theta)>0$ for all $\theta>0$. Fig. 7 shows a full rotation of (15), and Fig. 8 shows a plot of the curvature for a full rotation with an asterisk indicating the value of $\theta$ corresponding to the maximum curvature. The curve was first studied by Fermat in 1636 and published in (Fermat, 1679).

\subsection{Cartesian Parametrization}

A Cartesian parametrization of (15) is (Dahl, 2013):

$$
\left[\begin{array}{l}
x \\
y
\end{array}\right]=\left[\begin{array}{c}
r \cos (\theta) \\
r \sin (\theta)
\end{array}\right]=\left[\begin{array}{c}
k \sqrt{\theta} \cos (\theta) \\
k \sqrt{\theta} \sin (\theta)
\end{array}\right] .
$$




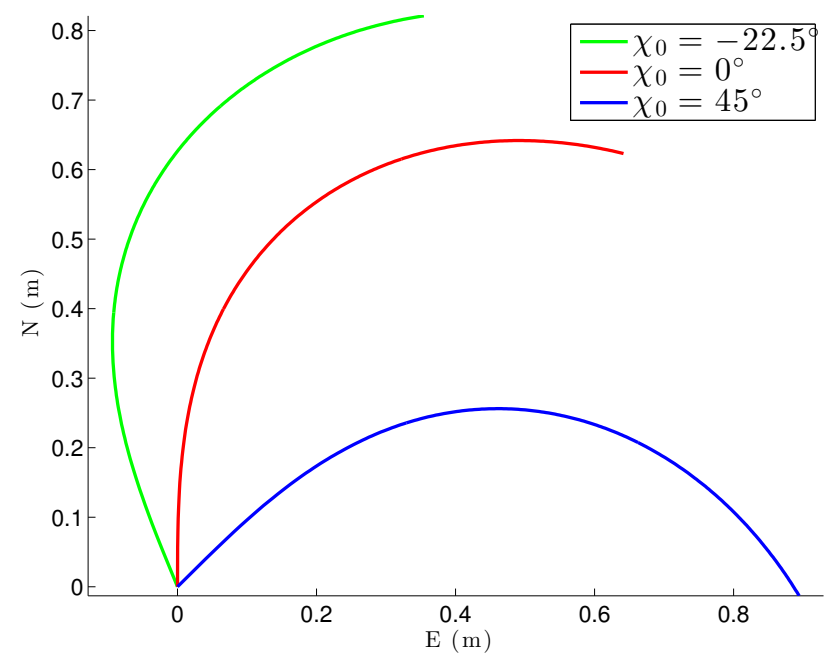

Figure 9: Fermat's spiral segment with fixed scaling $(\mathrm{k}=1)$ and different initial tangent angles

To allow for different initial positions $\mathbf{p}_{0}=\left[x_{0}, y_{0}\right]^{\mathrm{T}}$, turning both left and right, and different initial tangent angles $\chi_{0}$, the following parametrization is proposed:

$$
\mathbf{p}_{\mathrm{FS}}(\theta)=\left[\begin{array}{c}
x_{0}+k \sqrt{\theta} \cos \left(\rho \theta+\chi_{0}\right) \\
y_{0}+k \sqrt{\theta} \sin \left(\rho \theta+\chi_{0}\right)
\end{array}\right],
$$

where

$$
\rho=\left\{\begin{aligned}
1 & \text { for an anti-clockwise turn, and } \\
-1 & \text { for a clockwise turn. }
\end{aligned}\right.
$$

The domain in $(18)$ is $\theta \in\left[0, \theta_{\text {end }}\right]$, where $\theta_{\text {end }}$ is the parameter corresponding to the spiral end point, yet to be determined. This parametrization is thus neither unit domain nor unit speed, but it can be made unit domain by setting $\theta=\varpi \theta_{\text {end }}$. Fig. 9 shows how changing $\chi_{0}$ affects the orientation of the spiral and Fig. 10 demonstrates the effect of the scaling constant $k$.

As was the case with the clothoid in Sec. 2.4, we will distinguish between FS smoothing and circular smoothing with FS transition. This implies that a mirrored version of (18) is needed to describe a curve of curvature decreasing to zero. The following is proposed:

$$
\begin{aligned}
& \mathbf{p}_{\overline{\mathrm{FS}}}(\theta)= \\
& \quad\left[\begin{array}{c}
x_{\text {end }}+k \sqrt{\theta_{\text {end }}-\theta} \cos \left(\rho\left(\theta-\theta_{\text {end }}\right)+\chi_{\text {end }}\right) \\
y_{\text {end }}+k \sqrt{\theta_{\text {end }}-\theta} \sin \left(\rho\left(\theta-\theta_{\text {end }}\right)+\chi_{\text {end }}\right)
\end{array}\right],
\end{aligned}
$$

where $\mathbf{p}_{\text {end }}=\left[x_{\text {end }}, y_{\text {end }}\right]^{\mathrm{T}}$ is the position at the end of the curve, i.e. $\mathbf{p}_{\text {end }}=\mathbf{p}_{\overline{\mathrm{FS}}}\left(\theta_{\mathrm{end}}\right)$, and $\chi_{\mathrm{end}}$ is the course angle at that point.

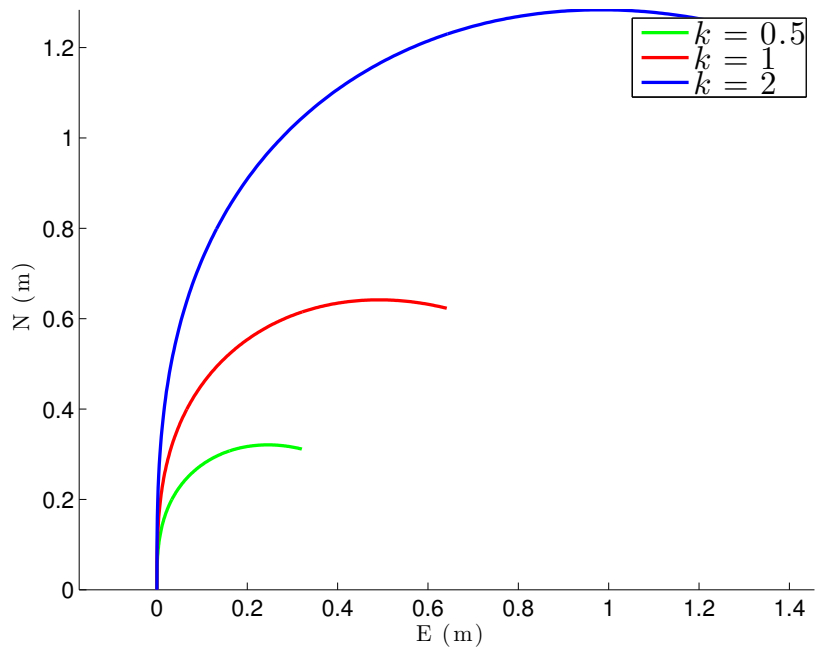

Figure 10: Fermat's spiral with fixed initial tangent angle $\left(\chi_{0}=0\right)$ and different scaling constants

\subsection{Parametrized Speed and Acceleration}

The first derivative of (18) is (Dahl, 2013):

$$
\begin{aligned}
& \frac{d}{d \theta} \mathbf{p}_{\mathrm{FS}}(\theta)= \\
& \frac{k}{2 \sqrt{\theta}}\left[\begin{array}{c}
\cos \left(\rho \theta+\chi_{0}\right)-2 \rho \theta \sin \left(\rho \theta+\chi_{0}\right) \\
\sin \left(\rho \theta+\chi_{0}\right)+2 \rho \theta \cos \left(\rho \theta+\chi_{0}\right)
\end{array}\right]
\end{aligned}
$$

and the second derivative is

$$
\begin{aligned}
& \frac{d^{2}}{d \theta^{2}} \mathbf{p}_{\mathrm{FS}}(\theta)= \\
& \frac{-k}{4 \theta^{\frac{3}{2}}}\left[\begin{array}{r}
\left(4 \theta^{2}+1\right) \cos \left(\rho \theta+\chi_{0}\right)+4 \rho \theta \sin \left(\rho \theta+\chi_{0}\right) \\
\left(4 \theta^{2}+1\right) \sin \left(\rho \theta+\chi_{0}\right)-4 \rho \theta \cos \left(\rho \theta+\chi_{0}\right)
\end{array}\right] .
\end{aligned}
$$

Similarly, for the mirrored curve (20), the first and second derivatives are given by (34)-(35). Equations (21)-(22) and (34)-(35) have a singularity at the beginning of the path, i.e. $\theta=0$. This is a drawback since the velocity and acceleration at the beginning and end of the path are undefined, a property that makes the Fermat spiral segment initially appear unsuitable for path-tracking applications in its current form. However, this is a property of the parametrization (that is, the kinematics and not the geometry of the curve) and it is possible to remedy the problem by changing variables, as was discussed by Peters (2012). Given that the problem is caused by the term $\sqrt{\theta}$ in the denominator and that the Cartesian parametrization of the FS resembles that of a circle, we change variables as follows:

$$
u:=\sqrt{\theta} \Rightarrow 0 \leq u \leq \sqrt{\theta_{\max }}
$$




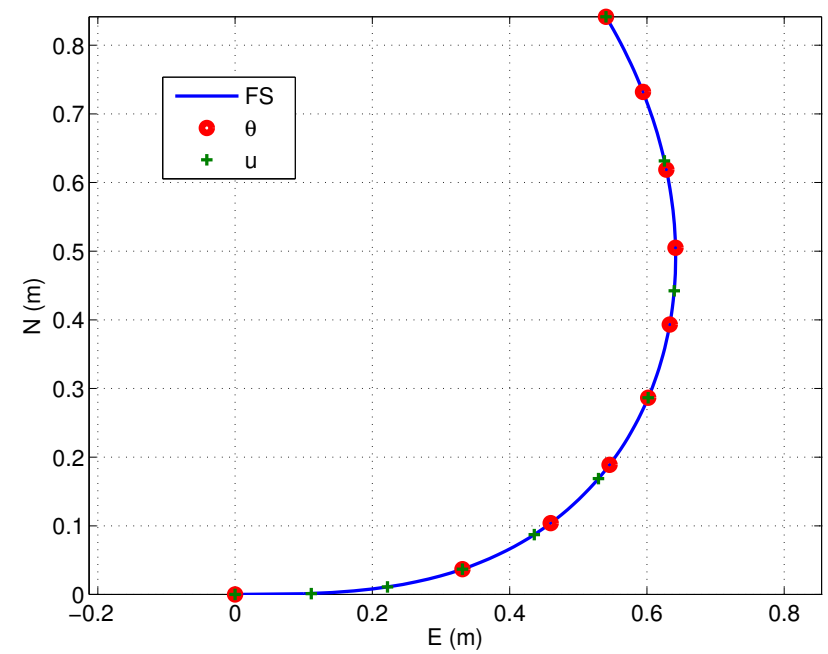

Figure 11: Illustration of the two different parametrizations. The geometry of the curve remains unaffected.

and then (18) is written:

$$
\mathbf{p}_{\mathrm{FS}}(u)=\left[\begin{array}{c}
x_{0}+k u \cos \left(\rho u^{2}+\chi_{0}\right) \\
y_{0}+k u \sin \left(\rho u^{2}+\chi_{0}\right)
\end{array}\right],
$$

which gives the singularity-free expression for the velocity:

$$
\begin{aligned}
& \frac{d}{d u} \mathbf{p}_{\mathrm{FS}}(u)= \\
& \quad k\left[\begin{array}{c}
\cos \left(\rho u^{2}+\chi_{0}\right)-2 \rho u^{2} \sin \left(\rho u^{2}+\chi_{0}\right) \\
\sin \left(\rho u^{2}+\chi_{0}\right)+2 \rho u^{2} \cos \left(\rho u^{2}+\chi_{0}\right)
\end{array}\right] .
\end{aligned}
$$

It is necessary to show that (24) results in a regular curve. To this end, we employ the amplitude-phase expressions

$$
\begin{aligned}
a \sin (x)+b \cos (x) & =\sqrt{a^{2}+b^{2}} \sin (x+\phi), \\
a \cos (x)+b \sin (x) & =\sqrt{a^{2}+b^{2}} \sin (x-\phi), \\
\phi & =\operatorname{atan} 2(b, a),
\end{aligned}
$$

and rewrite (25) as:

$$
\begin{aligned}
& \frac{d}{d u} \mathbf{p}_{\mathrm{FS}}(u)= \\
& \quad k \sqrt{1+\left(2 \rho u^{2}\right)^{2}}\left[\begin{array}{c}
\sin \left(\rho u^{2}+\chi_{0}-\phi\right) \\
\cos \left(\rho u^{2}+\chi_{0}-\phi\right)
\end{array}\right],
\end{aligned}
$$

with $\phi=\operatorname{atan} 2\left(1,4 \rho^{2} u^{4}\right)$. For a regular curve it is required that:

$$
\left|\frac{d}{d u} \mathbf{p}_{\mathrm{FS}}(u)\right| \neq 0
$$

For (29) we have that:

$$
\left|\frac{d}{d u} \mathbf{p}_{\mathrm{FS}}(u)\right|=k \sqrt{2} \sqrt{1+4 \rho^{2} u^{4}}
$$

which guarantees that (30) is satisfied. As a consequence, the FS can be used for path-tracking applications. In that case, the derivative of the parameter w.r.t time has to be defined. According to Breivik and Fossen (2004a) and (Fossen, 2011, Sec. 10.4), for a desired vehicle speed $U_{d}(t)$, the parameter derivative w.r.t time is determined by:

$$
\dot{u}(t)=\frac{U_{d}(t)}{\sqrt{x_{p}^{\prime}(u)^{2}+y_{p}^{\prime}(u)^{2}}},
$$

and from (31)-(32):

$$
\dot{u}=\frac{U_{d}}{k \sqrt{2} \sqrt{1+4 u^{4}}},
$$

where we used that $\rho^{2}=1$. Fig. 11 shows the image of an FS segment when each of the parameters $\theta$ and $u$ is sampled at 10 equispaced points.

\subsection{Path Length}

One, often important, consideration when designing paths is the length of the path, an example can be found in (Jørgensen and Skjetne, 2012). Before moving on, it should be noted that we assume no external

$$
\begin{gathered}
\frac{d}{d \theta} \mathbf{p}_{\overline{\mathrm{FS}}}(\theta)=-\frac{k}{2 \sqrt{\theta_{\text {end }}-\theta}}\left[\begin{array}{c}
\cos \left(\rho\left(\theta-\theta_{\text {end }}\right)+\chi_{0}\right)+2 \rho\left(\theta_{\text {end }}-\theta\right) \sin \left(\rho\left(\theta-\theta_{\text {end }}\right)+\chi_{0}\right) \\
\sin \left(\rho\left(\theta-\theta_{\text {end }}\right)+\chi_{0}\right)+2 \rho\left(\theta_{\text {end }}-\theta\right) \cos \left(\rho\left(\theta-\theta_{\text {end }}\right)+\chi_{0}\right)
\end{array}\right] \\
\frac{d^{2}}{d \theta^{2}} \mathbf{p}_{\overline{\mathrm{FS}}}(\theta)= \\
-\frac{k}{4\left(\theta_{\text {end }}-\theta\right)^{\frac{3}{2}}}\left[\begin{array}{l}
\left(4\left(\theta-\theta_{\text {end }}\right)^{2}+1\right) \cos \left(\rho\left(\theta-\theta_{\text {end }}\right)+\chi_{0}\right)-4 \rho\left(\theta_{\text {end }}-\theta\right) \sin \left(\rho\left(\theta-\theta_{\text {end }}\right)+\chi_{0}\right) \\
\left.4\left(\theta-\theta_{\text {end }}\right)^{2}+1\right) \sin \left(\rho\left(\theta-\theta_{\text {end }}\right)+\chi_{0}\right)+4 \rho\left(\theta_{\text {end }}-\theta\right) \cos \left(\rho\left(\theta-\theta_{\text {end }}\right)+\chi_{0}\right)
\end{array}\right]
\end{gathered}
$$


disturbances acting on the vehicle, therefore the path length is discussed here from a geometric point of view. As mentioned in Sec. 1, the Dubins path is the shortest one between two poses in a plane if we consider a unit speed vehicle with bounded curvature (alternatively, turning radius). Calculating the total length of a Dubins path is trivial since the expressions for the length of straight lines and circular arcs are well known and easy to compute.

Despite their complex Cartesian parametrization, the clothoids have the nice property of a curvature linearly varying w.r.t. the path parameter. As a result, there is a linear relationship between the curvature and the clothoid arc length, meaning that the clothoid arc length is easy to compute.

Computing the length of an FS arc is not as trivial as the aforementioned cases because (using the parametrization of Sec. 3.3) it is given by the integral:

$$
L_{\mathrm{FS}}=k \sqrt{2} \int_{0}^{u_{\max }} \sqrt{1+4 u^{4}} d u,
$$

which has no closed-form solution. The path length can be expressed as a Gaussian hypergeometric function:

$$
L_{\mathrm{FS}}(\theta)=k \sqrt{\theta}{ }_{2} F_{1}\left(-\frac{1}{2}, \frac{1}{4} ; \frac{5}{4} ;-4 \theta^{2}\right),
$$

see (Weisstein, 2013b). It should be noted that, using the parametrization of Sec. 3.3, (37) is equivalent to:

$$
L_{\mathrm{FS}}(u)=k u_{2} F_{1}\left(-\frac{1}{2}, \frac{1}{4} ; \frac{5}{4} ;-4 u^{4}\right) .
$$

According to (Abramowitz and Stegun, 1970, Ch. 15), the circle of convergence of the Gauss hypergeometric series

$$
{ }_{2} F_{1}(a, b ; c ; z)=\sum_{n=0}^{\infty} \frac{(a)_{n}(b)_{n}}{(c)_{n}} \frac{z^{n}}{n !}
$$

is the unit circle $|z|<1$, which corresponds to $\theta<1 / 2$, or a course change $\Delta \chi<73.6479^{\circ}$. The behavior of the series (39) on its circle of convergence is absolute convergence when $\operatorname{Re}(c-a-b)>0$, which is true for (37)-(38) since $5 / 4+1 / 2-1 / 4=3 / 2$. The closer to zero $z$ is, the faster and more accurate the computation of (39) becomes.

The restriction mentioned above regarding the circle of convergence does not forbid us to compute the length of an FS arc for course changes equal to and higher than $73.6479^{\circ}$. As it can be seen in (Pearson, 2009, Sec. 4.6), for $z \in \mathbb{R}$ it is possible to use appropriate transformation formulae in order to map $z$ to a new variable $w \in[0,1 / 2]$, hence resulting in a fast and accurate computation for all $z$, and thus $\theta$.

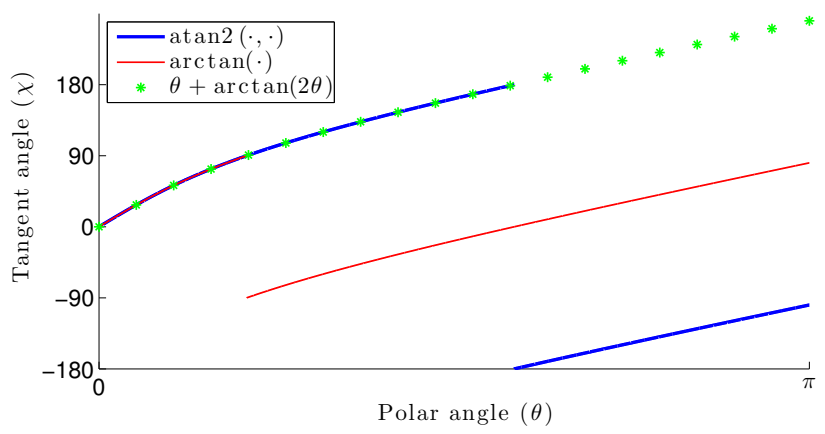

Figure 12: Course tangent angle. Only the expression $\theta+\arctan (2 \theta)$ is continuous.

To conclude, the computation of the length of an FS arc is not as straightforward as that of a circular, or even clothoid, arc. On the positive side, the FS length is given by a hypergeometric series that always converges and is easy to compute.

\subsection{Course Angle}

The course, or direction, along the curve can be determined by (6). It is well-defined since the singular terms of (21) cancel. Moreover (Dahl, 2013):

$$
\chi(\theta)=\arctan \left(\frac{\sin (\theta)+2 \theta \cos (\theta)}{\cos (\theta)-2 \theta \sin (\theta)}\right),
$$

or, expressed with the two-argument arctangent function,

$$
\chi(\theta)=\arctan 2\left(\begin{array}{c}
\sin (\theta)+2 \theta \cos (\theta) \\
\cos (\theta)-2 \theta \sin (\theta)
\end{array}\right) .
$$

We rewrite (40) as follows:

$$
\begin{aligned}
\chi(\theta) & =\int\left(\frac{d}{d \theta} \arctan \left(\frac{\sin (\theta)+2 \theta \cos (\theta)}{\cos (\theta)-2 \theta \sin (\theta)}\right)\right) d \theta \\
& =\int \frac{4 \theta^{2}+3}{4 \theta^{2}+1} d \theta
\end{aligned}
$$

where, finally, the equations are continuous and equal to:

$$
\chi(\theta)=\theta+\arctan (2 \theta)
$$

up to respectively $\chi=90^{\circ}$ and $\chi=180^{\circ}$. The three expressions (40)-(41) and (43) are plotted in Fig. 12 for comparison. In practice, a $180^{\circ}$ course change is considered sufficient for one curve because a full rotation is achieved when combined with a mirrored curve. 


\section{Fermat Spiral Path Design}

\subsection{Problem Description}

In this section we present a methodology for using the FS in order to construct curvature-continuous paths. Given a piecewise linear path, the vertices can be smoothened by two Fermat curves, one entering and one exiting (mirrored) curve. The vertices then become curvature continuous $\left(G^{2}\right)$ and respect a given curvature constraint $\kappa_{\max }$.

Without loss of generality, we assume the initial piecewise linear path consists of two straight lines connecting three sequential waypoints $\left(\mathbf{w} \mathbf{p t} \mathbf{t}_{i-1}, \mathbf{w} \mathbf{p t} \mathbf{t}_{i}, \mathbf{w} \mathbf{p t} \mathbf{t}_{i+1}\right)$ on the plane, see Fig. 13. Initially, the course change direction $\rho$ must be determined, for this reason it is useful to define two normalized vectors parallel to the straight lines (see Fig. 14):

$$
\begin{aligned}
\mathbf{v}_{\text {in }} & =\frac{\mathbf{w p t}_{i}-\mathbf{w} \mathbf{p} \mathbf{t}_{i-1}}{\left\|\mathbf{w} \mathbf{p} \mathbf{t}_{i}-\mathbf{w} \mathbf{p} \mathbf{t}_{i-1}\right\|}, \\
\mathbf{v}_{\text {out }} & =\frac{\mathbf{w p t}_{i+1}-\mathbf{w} \mathbf{p} \mathbf{t}_{i}}{\left\|\mathbf{w} \mathbf{p} \mathbf{t}_{i+1}-\mathbf{w} \mathbf{p} \mathbf{t}_{i}\right\|} .
\end{aligned}
$$

Then, the course change magnitude can be computed as follows:

$$
|\Delta \chi|=\arccos \left(\mathbf{v}_{\text {in }} \cdot \mathbf{v}_{\text {out }}\right),
$$

and the course change direction:

$$
\rho=-\operatorname{sign}\left(\mathrm{v}_{\text {in }, \mathrm{y}} \mathrm{v}_{\text {out }, \mathrm{x}}-\mathrm{v}_{\text {in }, \mathrm{x}} \mathrm{v}_{\text {out }, \mathrm{y}}\right) .
$$

\subsection{Domain Determination}

The domain of $\theta$ in (18) must be set according to the desired course change from the beginning to the end of the curve. Similarly, the scaling constant $k$ must be set to respect the curvature constraint $\kappa_{\max }$.

The domain's upper limit $\theta_{\text {end }}$ determines the course change. Unfortunately, (43) is not invertible, thus it is not possible to determine the $\theta_{\text {end }}$ needed to achieve a desired course $\chi$ analytically.

However, (43) is continuous and differentiable, even beyond $\chi=180^{\circ}$ and numerical solutions are therefore possible. This problem was investigated in (Dahl, 2013) where it was concluded that Halley's method (or, equivalently, Householder's method of order 2), given by:

$$
x_{n+1}=x_{n}-\frac{2 f\left(x_{n}\right) f^{\prime}\left(x_{n}\right)}{2\left(f^{\prime}\left(x_{n}\right)\right)^{2}-f\left(x_{n}\right) f^{\prime \prime}\left(x_{n}\right)},
$$

was the most efficient. More specifically, Halley's method was sufficiently efficient for tolerance equal to $10^{-3}$ after only one step.

\subsection{Scaling Determination}

Once the domain of (18) is determined, the scaling constant $k$ must be set such that the curvature constraint $\kappa_{\max }$ is satisfied all over the curve. As given by (16), the curvature reaches its maximum at:

$$
\theta=\sqrt{\frac{\sqrt{7}}{2}-\frac{5}{4}}
$$

In the case where the domain does not include this point, the maximum curvature is at $\theta_{\text {end }}$. Thus, the parameter corresponding to the maximum curvature is

$$
\theta_{\kappa_{\max }}=\min \left(\theta_{\text {end }}, \sqrt{\frac{\sqrt{7}}{2}-\frac{5}{4}}\right) .
$$

Solving (16) for $k$ yields that, in order for the maximum curvature along the curve to be the same as the curvature constraint, i.e. $\kappa\left(\theta_{\kappa_{\max }}\right)=\kappa_{\max }$, it is necessary with

$$
k=\frac{1}{\kappa_{\max }} \frac{2 \sqrt{\theta_{\kappa_{\max }}}\left(3+4 \theta_{\kappa_{\max }}^{2}\right)}{\left(1+4 \theta_{\kappa_{\max }}^{2}\right)^{\frac{3}{2}}} .
$$

\subsection{Length and Height Calculations}

The projected length $l_{1}$ of the FS curve along the initial course and the height $h$ perpendicular to it are useful for determining the initial position $\mathbf{p}_{0}$. Both are found by considering (18) with $\chi_{0}=0$. The length is then the $x$-component (see Fig. 15):

$$
l_{1}=k \sqrt{\theta_{\text {end }}} \cos \left(\theta_{\text {end }}\right),
$$

and the height is the $y$-component (see Fig. 16):

$$
h=k \sqrt{\theta_{\text {end }}} \sin \left(\theta_{\text {end }}\right) .
$$

\subsection{Fermat Smoothing}

The initial and final course angles, $\chi_{0}$ and $\chi_{\text {end }}$, can be determined in many ways. For instance, given tangent

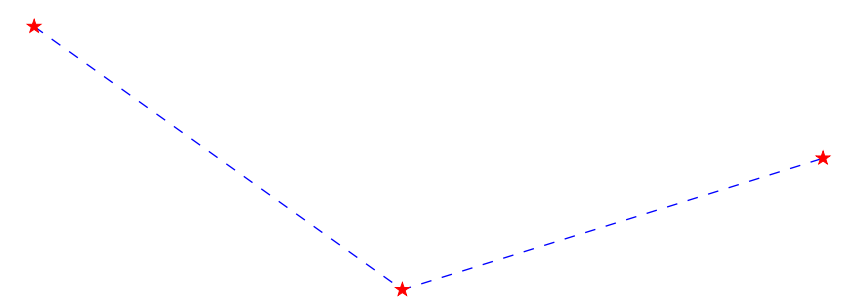

Figure 13: Initial path of two straight lines connecting three sequential waypoints on the plane 


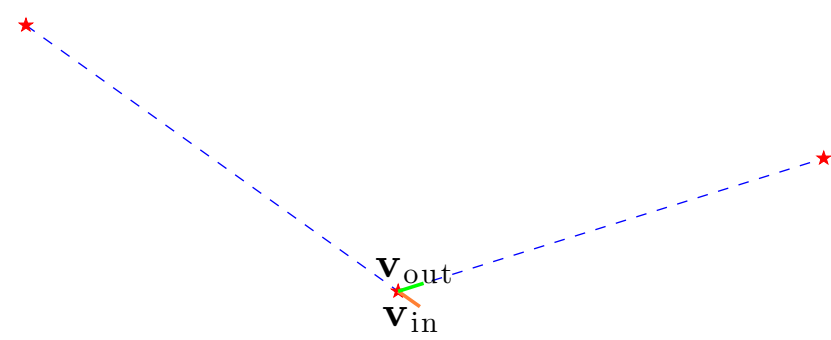

Figure 14: Two normalized vectors parallel to the straight lines are defined
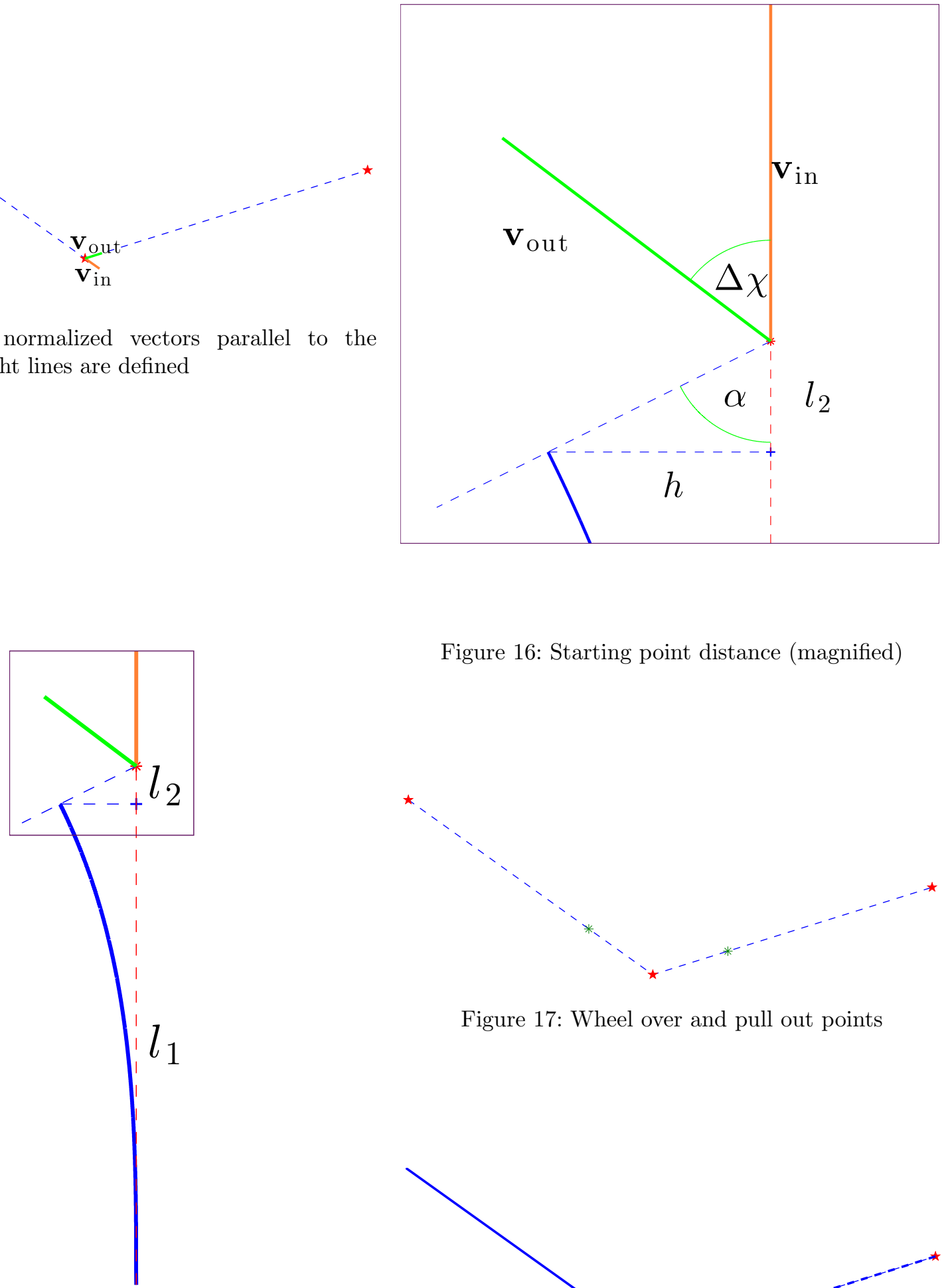

Figure 16: Starting point distance (magnified)

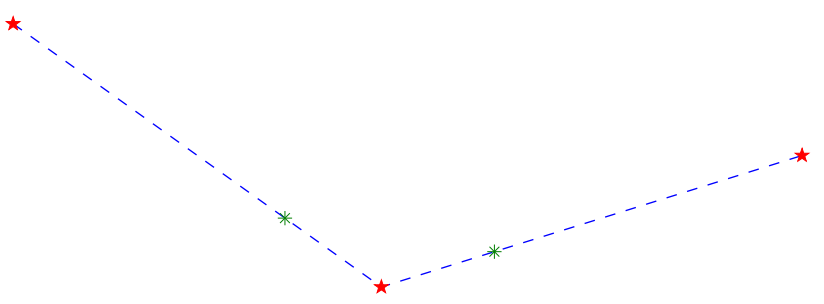

Figure 17: Wheel over and pull out points

Figure 15: Starting point distance

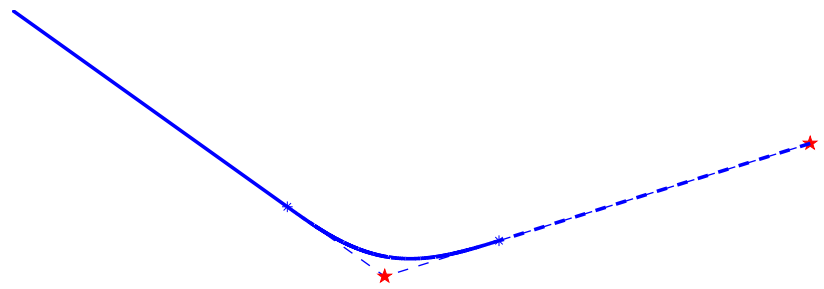

Figure 18: Final path 


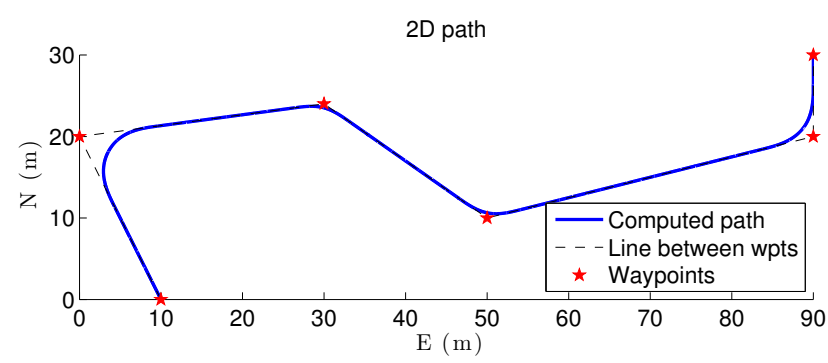

Figure 19: Fermat-smoothened path
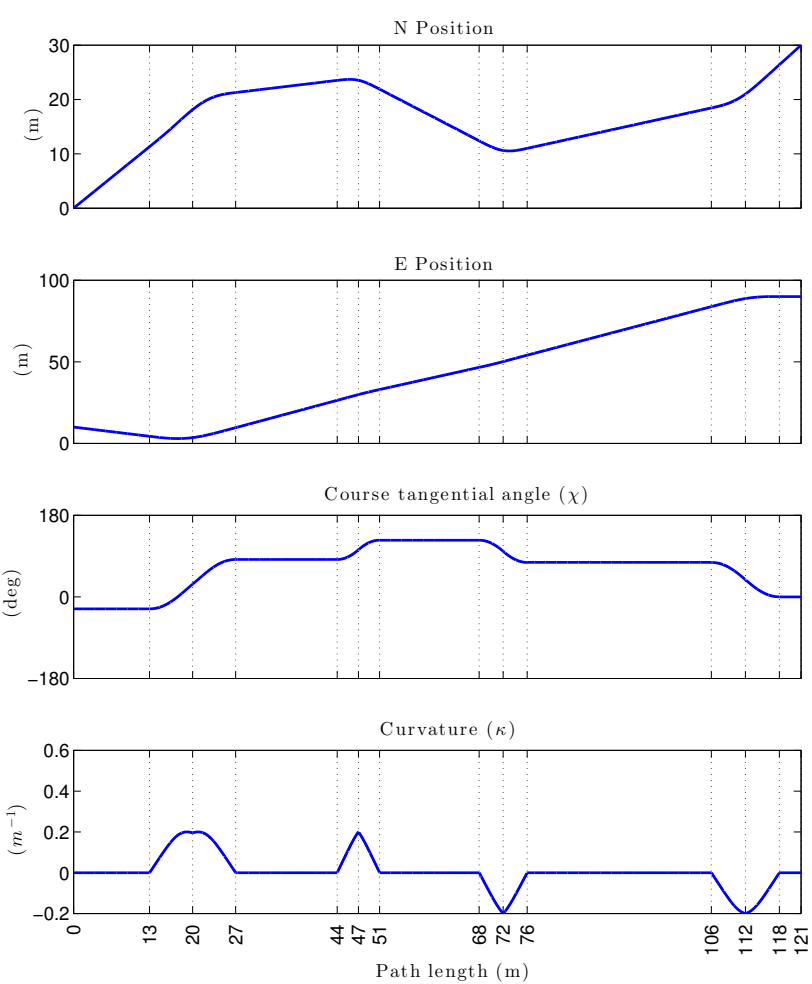

Figure 20: Fermat smoothing path properties

vectors:

$$
\begin{aligned}
\chi_{0} & =\operatorname{atan} 2\left(\mathrm{v}_{\text {in }, \mathrm{y}}, \mathrm{v}_{\text {in }, \mathrm{x}}\right), \\
\chi_{\text {end }} & =\operatorname{atan} 2\left(\mathrm{v}_{\text {out }, \mathrm{y}}, \mathrm{v}_{\text {out }, \mathrm{x}}\right) .
\end{aligned}
$$

Finally, the initial and final positions $\mathbf{p}_{0}$ and $\mathbf{p}_{\text {end }}$ must be determined such that the curves meet. Fig. 15 shows that these must be at a distance

$$
l=l_{1}+l_{2}
$$

along the linear path from the waypoint. The distance $l_{1}$ is given by (52). From the figure it can be seen that

$$
l_{2}=\frac{h}{\tan (\alpha)}
$$

where $h$ is given by (53) and

$$
\alpha=\frac{180^{\circ}-\Delta \chi}{2},
$$

as seen from Fig. 16. There is no danger for the angle $\alpha$ being equal to zero, and hence inducing a singularity at Eq. (57). This would imply a course change of $180^{\circ}$, which will never occur for two successive waypoints. Figs 17-18 show the wheel over and pull out points, and the FS arc connecting them, respectively.

Figs 19-20 show the Fermat smoothing path and its properties. Similarly to the clothoid, due to the numeric solution of $\theta_{\text {end }}$, the apparent continuity is not true: a gap exists at the transition between the Fermat curves. Still, any numeric precision can be achieved, consequently making the discontinuity correspondingly small and practically neglectable. Except for this, the path is visibly both tangent and curvature continuous, thus $G^{2}$.

It should be noted that the path curvature is $\kappa_{\max }$ at two points, unless the required course change is $\Delta \chi \leq 43.83^{\circ 1}$. However, it is more reasonable to stay at maximum curvature for as long as possible once reaching it. Such a path is discussed in Sec. 4.6.

\subsection{Dubins Path with Fermat Transition}

As mentioned earlier, in addition to using FS for connecting straight paths, FS can be used as a transition curve between a straight line and a circular arc. In this way, the resulting path can be the same as a Dubins path with the exception of the transitional FS segment. A similar approach where the clothoid was used as the transition curve was proposed in (Fraichard and Scheuer, 2004). A Dubins path with FS transition and its properties can be seen in Figs 21-22. The curvature plot shows a more realistic and attractive behavior, compared to that of the previous section. Similarly to the original Dubins path case, this is an interpolating path.

\section{Fermat Spiral Path Evaluation}

In (Lekkas and Fossen, 2013) and (Dahl, 2013), the authors developed a number of path evaluation criteria that can be used for comparing paths depending on the application. Those criteria were:

\section{- Smoothness}

${ }^{1}$ When $\theta_{\text {end }}>\sqrt{\frac{\sqrt{7}}{2}-\frac{5}{4}}=0.27$, the curvature $\kappa_{\max }$ is reached at $\theta=\sqrt{\frac{\sqrt{7}}{2}-\frac{5}{4}}$ both on the entering and exiting curve. When $\theta_{\text {end }} \leq \sqrt{\frac{\sqrt{7}}{2}-\frac{5}{4}}$, the curvature $\kappa_{\text {max }}$ is reached only at the transition between the curves. 


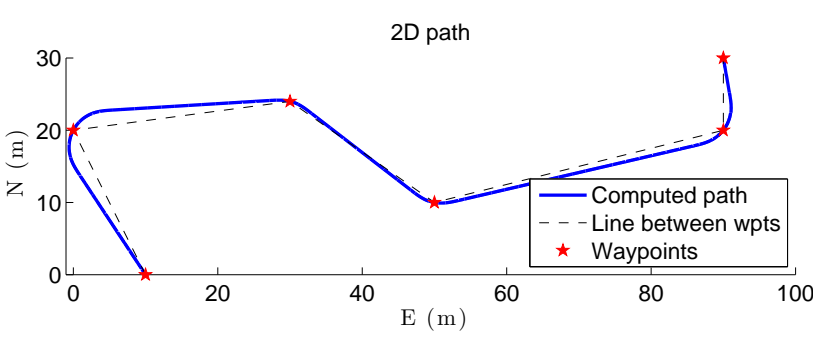

Figure 21: Dubins path with Fermat transition
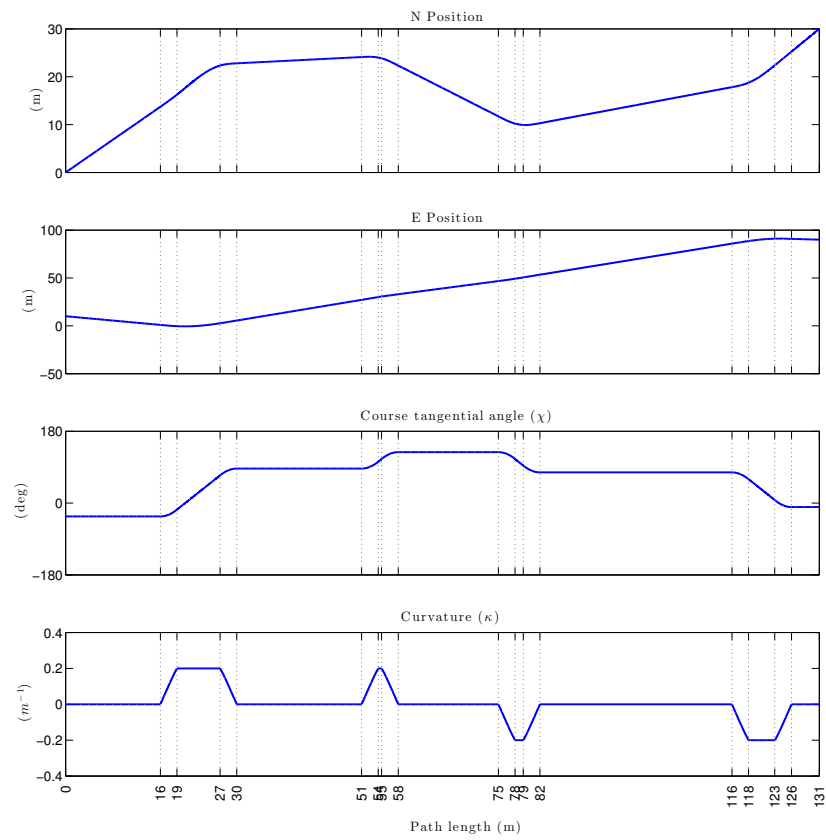

Figure 22: Dubins path with Fermat smoothing properties

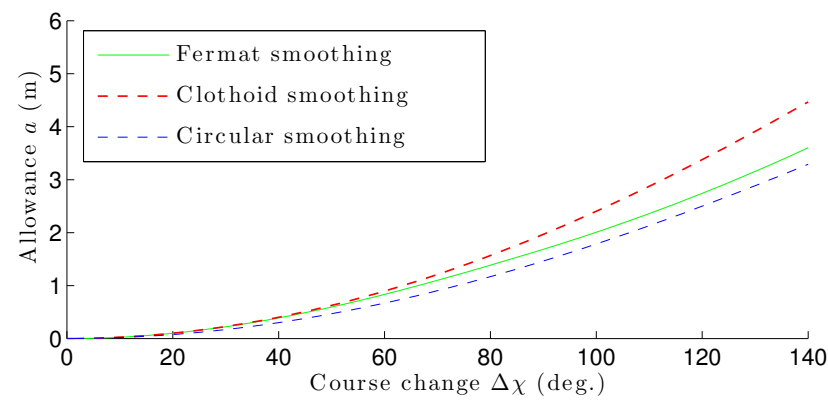

Figure 23: Fermat, clothoid and circular smoothing allowance comparison
- Length

- Allowance

- Tractability

- Algorithm Complexity

Regarding the FS smoothing path, we conclude the following:

Smoothness: The path has a continuous curvature and, in addition, it was shown in Sec. 3.3 that a different parametrization results in parametric continuity as well. Table 1 shows how the FS path compares with the other paths considered in this paper, when it comes to geometric continuity. Note that this is true for both interpolating and approximating versions of the path.

Length: The length of an FS arc can be computed by a hypergeometric series which converges for any value of $\theta$, as it was shown in Sec. 3.4. Naturally, a path consisting of straight lines and FS segments will be longer than a Dubins path.

Allowance: Allowance refers to how much the smoothing arc deviates from the initial piecewise linear path. This can be useful when the vehicle navigates in an area with obstacles. In this case the allowance is computed as:

$$
a=k \sqrt{\theta_{\text {end }}} \sin \left(\theta_{\text {end }}\right) .
$$

Fig. 23 shows the allowance $a$ for different course change magnitudes $\Delta \chi$. The comparison includes the FS, the clothoid and the circular arc. It can be observed that the Fermat smoothing allowance is almost the same as that of the clothoid for course changes up to $30^{\circ}$. Above that value, the FS allowance is smaller than the clothoid's, and always larger than that of the circle.

Tractability: The effect of moving a waypoint is the same as for the clothoid, resulting in local influence, which means that if one waypoint changes location only the neighboring path segments will be affected. This is a useful property when a vehicle navigates in an area with obstacles and a waypoint has to be relocated during operation, for instance, due to weather conditions.

Algorithm Complexity: The process of computing an FS is significantly less computationally expensive than the clothoid. The exact computational times will vary (depending on the implementation platform), but FS involves only the numerical solution of (43), while the clothoid requires calculating the Fresnel integrals. 


\begin{tabular}{lccc}
\hline Method & $G^{0} / C^{0}$ & $G^{1}$ & $G^{2}$ \\
\hline Piecewise linear & $\checkmark$ & $x$ & $x$ \\
\hline Circular arc smoothing & $\checkmark$ & $\checkmark$ & $x$ \\
Clothoid smoothing & $\checkmark$ & $\checkmark$ & $\checkmark$ \\
Fermat smoothing & $\checkmark$ & $\checkmark$ & $\checkmark$ \\
\hline
\end{tabular}

Table 1: Geometric continuity comparison

\section{Conclusions}

This work has considered the development of a new methodology, based on Fermat's spiral (FS), for generating curvature-continuous paths that can be used for path-following and path-tracking applications for underactuated vehicles. The main motivation has been to construct paths of simple geometry (preferably consisting of straight lines and arc segments) which avoid the curvature discontinuity of Dubins paths and the computational intensity of the clothoids. The FS was considered as a good candidate because it has a zero curvature at its origin, hence making it possible to connect it smoothly with straight lines. The properties of the FS have been explored and an alternative parametrization has been proposed for guaranteeing parametric speed continuity, an important constraint when it comes to path-tracking applications. Despite the fact that the parameter end point (depending on the course change between two sequential straight lines) can only be computed numerically, the computational load is much lighter than that of the clothoid.

Further work includes a generalization to $3 \mathrm{D}$ paths and the design of parallel FS paths for formation control of multiple vehicles.

\section{Acknowledgments}

This work was supported by the Norwegian Research Council through the Centre for Ships and Ocean Structures (CeSOS, project number 146025) as well as the Centre for Autonomous Marine Operations and Systems (AMOS, project number 223254) at NTNU, and through the MAROFF project "D2V" (project number 210670).

\section{References}

Abramowitz, M. and Stegun, I. Handbook of mathematical functions. Dover Publishing Inc. New York, 1970.

Bakolas, E. and Tsiotras, P. Time-optimal synthesis for the Zermelo-Markov-Dubins problem: The constant wind case. In American Control Conference. IEEE, Baltimore, Maryland, USA, 2010.

Bakolas, E. and Tsiotras, P. Optimal synthesis of the Zermelo-Markov-Dubins problem in a constant drift field. Journal of Optimization Theory and Applications, 2013. 156(2):469-492. doi:10.1007/s10957012-0128-0.

Barsky, B. A. and DeRose, T. D. Geometric continuity of parametric curves: three equivalent characterizations. IEEE Computer Graphics and Applications, 1989. 9(6):60-69. doi:10.1109/38.41470.

Breivik, M. and Fossen, T. I. Path following for marine surface vessels. In Proceedings of the OTO'04. Kobe, Japan, 2004a. doi:10.1109/OCEANS.2004.1406507.

Breivik, M. and Fossen, T. I. Path following of straight lines and circles for marine surface vessels. In Proceedings of the IFAC Conference on Control Applications in Marine Systems. Ancona, Italy, 2004b.

Breivik, M. and Fossen, T. I. Guidance laws for autonomous underwater vehicles, chapter 4, pages 5176. INTECH Education and Publishing, 2009.

Bruyninckx, H. and Reynaerts, D. Path planning for mobile and hyper-redundant robots using Pythagorean hodograph curves. In Proceedings of the 8th International Conference on Advanced Robotics. IEEE, Monterey, CA, USA, 1997. doi:10.1109/ICAR.1997.620243.

Candeloro, M., Lekkas, A. M., Sørensen, A. J., and Fossen, T. I. Continuous curvature path planning using Voronoi diagrams and Fermat's spirals. In 9th IFAC Conference on Control Applications in Marine Systems. Osaka, Japan, 2013.

Chang, A. J., Brazil, M., Rubinstein, J. H., and Thomas, D. A. Curvature-constrained directionalcost paths in the plane. Journal of Global Optimization, 2012. 53(4):663-681. doi:10.1007/s10898-0119730-1.

Chitsaz, H. and LaValle, S. M. Time-optimal paths for a Dubins airplane. In Proceedings of the 46th IEEE Conference on Decision and Control. New Orleans, Louisiana, USA, 2007. doi:10.1109/CDC.2007.4434966.

Dahl, A. R. Path Planning and Guidance for Marine Surface Vessels. Master's thesis, Norwegian University of Science and Technology, 2013.

Dubins, L. E. On curves of minimal length with a constraint on average curvature, and with prescribed 
initial and terminal positions and tangents. American Journal of Mathematics, 1957. 79(3):497-516. doi: $10.2307 / 2372560$.

Eriksson-Bique, S., Kirkpatrick, D., and Polishchuk, V. Discrete Dubins paths. arXiv preprint arXiv:1211.2365, 2012.

Farin, G. and Sapidis, N. Curvature and the fairness of curves and surfaces. IEEE Computer Graphics and Applications, 1989. 9(2):52-57. doi:10.1109/38.19051.

Farouki, R. T. Pythagorean-hodograph quintic transition curves of monotone curvature. Computer-Aided Design, 1997. 29(9):601-606. doi:10.1016/S00104485(97)00004-3.

Farouki, R. T. Pythagorean-hodograph Curves. Springer, 2008. doi:10.1007/978-3-540-73398-0.

Farouki, R. T. and Sakkalis, T. Pythagorean hodographs. IBM Journal of Research and Development, 1990. 34(5):736-752. doi:10.1147/rd.345.0736.

Farouki, R. T. and Sakkalis, T. Real rational curves are not 'unit speed'. Computer Aided Geometric Design, 1991. 8(2):151-157. doi:10.1016/01678396(91)90040-I.

Fermat, P. Ad locos planos et solidos isagoge. Varia Opera Mathematica, 1679.

Fossen, T. I. Handbook of Marine Craft Hydrodynamics and Motion Control. John Wiley and Sons Ltd., 2011. doi:10.1002/9781119994138.

Fossen, T. I. and Lekkas, A. M. Direct and indirect adaptive integral line-of-sight path-following controllers for marine craft exposed to ocean currents. International Journal of Adaptive Control and Signal Processing, 2014. (submitted).

Fraichard, T. and Scheuer, A. From Reeds and Shepp's to continuous-curvature paths. IEEE Transactions on Robotics, 2004. 20(6):1025-1035. doi:10.1109/TRO.2004.833789.

Gajny, L., Bearee, R., Nyiri, E., and Gibaru, O. Path planning with $\mathrm{PH} G^{2}$ splines in $R^{2}$. In Proceedings of the 1st International Conference on Systems and Computer Science. IEEE, Lille, France, 2012. doi:10.1109/IConSCS.2012.6502455.

Harary, G. and Tal, A. 3D Euler spirals for 3D curve completion. Computational Geometry, 2012. 45(3):115-126. doi:10.1016/j.comgeo.2011.10.001.
Haugen, J. Guidance Algorithms for Planar Path-based Motion Control Scenarios. Master's thesis, Norwegian University of Science and Technology, 2010.

Hota, S. and Ghose, D. Optimal trajectory generation for convergence to a rectilinear path. Journal of Intelligent $\&$ Robotic Systems, 2013. pages 1-20.

Jørgensen, U. and Skjetne, R. Generating safe and equally long trajectories for multiple unmanned agents. In 20th Mediterranean Conference on Control $\&$ Automation (MED). IEEE, Barcelona, Spain, 2012. doi:10.1109/MED.2012.6265862.

LaValle, S. M. Planning algorithms. Cambridge university press, 2006. doi:10.1017/CBO9780511546877.

Lekkas, A. M. and Fossen, T. I. Line-of-Sight Guidance for Path Following of Marine Vehicles, chapter 5, in Advanced in Marine Robotics, pages 6392. LAP LAMBERT Academic Publishing (O. Gal, Ed.), 2013.

Pearson, J. W. Computation of hypergeometric functions. Master's thesis, University of Oxford, 2009.

Peters, J. Changing variables. IEEE Computer Graphics and Applications, 2012. 32(3):88-93. doi:10.1109/MCG.2012.51.

Reeds, J. A. and Shepp, L. A. Optimal paths for a car that goes both forwards and backwards. $P a$ cific Journal of Mathematics, 1990. 145(2):367-393. doi:10.2140/pjm.1990.145.367.

Shanmugavel, M., Tsourdos, A., White, B., and Zbikowski, R. Co-operative path planning of multiple UAVs using Dubins paths with clothoid arcs. Control Engineering Practice, 2010. 18(9):10841092. doi:10.1016/j.conengprac.2009.02.010.

Shanmugavel, M., Tsourdos, A., Zbikowski, R., and White, B. Path planning of multiple UAVs with clothoid curves in two dimensions. In Proceedings of the 17th IFAC Symposium on Automatic Control in Aerospace. Toulouse, France, 2007a.

Shanmugavel, M., Tsourdos, A., Zbikowski, R., and White, B. A. 3D path planning for multiple UAVs using Pythagorean hodograph curves. In $A I A A$ Guidance, Navigation, and Control Conference and Exhibit, Hilton Head, South Carolina. 2007b.

Techy, L. and Woolsey, C. A. Minimum-time path planning for unmanned aerial vehicles in steady uniform winds. Journal of Guidance, Control, and Dynamics, 2009. 32(6):1736-1746. doi:10.2514/1.44580. 
Toussaint, G. J., Basar, T., and Bullo, F. Motion planning for nonlinear underactuated vehicles using $H_{\infty}$ techniques. In Proceedings of the American Control Conference. IEEE, Arlington, Virginia, USA, 2001.

Tsourdos, A., White, B., and Shanmugavel, M. Cooperative path planning of unmanned aerial vehicles. Wiley Online Library, 2011.

Weisstein, E. W. "Archimedean spiral." From
MathWorld-A Wolfram Web Resource (accessed 03/12/2013). 2013a. URL http://mathworld. wolfram. com/ArchimedeanSpiral .html.

Weisstein, E. W. "Fermat's Spiral." From MathWorld-A Wolfram Web Resource (accessed 19/12/2013). 2013b. URL http://mathworld. wolfram.com/FermatsSpiral.html. 